\title{
ERRATUM
}

Jochen Vandekerkhove $\cdot$ Steven Declerck

Erik Jeppesen · José Maria Conde-Porcuna

Luc Brendonck · Luc De Meester

\section{Dormant propagule banks integrate spatio-temporal heterogeneity in cladoceran communities}

Published online: 30 June 2005

(C) Springer-Verlag 2005

\section{Oecologia (2005) 142:109-116}

Due to an unfortunate error braces were missing in Table 1. The correct table is shown below.

Table 1 Schematic overview of all samples taken from the active zooplankton community (AC) and from the dormant propagule bank (DPB) of the pond M13

The braces indicate which samples were combined to create composite samples. Numbers in brackets give the numbers of locations sampled in the different pond zones. LIT littoral zone, $L I T+$ downwind littoral zone, $L I T+$ upwind littoral zone, $P E L$ pelagic zone, $D$ daytime sample, $N$ night-time sample

\begin{tabular}{|c|c|c|c|c|c|c|}
\hline $\mathrm{AC}$ & 2000 & May & D & PEL (8) & \multirow{6}{*}{ INTRAYEAR } & \multirow{14}{*}{ INTEGRATED } \\
\hline $\mathrm{AC}$ & 2000 & June & $\mathrm{D}$ & PEL (8) & & \\
\hline $\mathrm{AC}$ & 2000 & July & $\mathrm{D}$ & PEL (8) & & \\
\hline $\mathrm{AC}$ & 2000 & August & D & PEL (8) & & \\
\hline $\mathrm{AC}$ & 2000 & September & D & PEL (8) & & \\
\hline $\mathrm{AC}$ & 2000 & October & $\mathrm{D}$ & PEL (8) & & \\
\hline $\mathrm{AC}$ & 1996 & July & $\mathrm{D}$ & PEL (4) & \multirow{5}{*}{ - INTERYEAR } & \\
\hline $\mathrm{AC}$ & 1997 & July & D & PEL (4) & & \\
\hline $\mathrm{AC}$ & 1998 & July & D & PEL (4) & & \\
\hline $\mathrm{AC}$ & 1999 & July & D & PEL (4) & & \\
\hline $\mathrm{AC}$ & 2000 & July & D & PEL (4) & & \\
\hline $\mathrm{AC}$ & 2000 & July & $\mathrm{N}$ & PEL (4) & $\rightarrow$ NIGHTPEL & \\
\hline $\mathrm{AC}$ & 2000 & July & D & PEL (4) & $\rightarrow$ DAYPEL & \\
\hline $\mathrm{AC}$ & 2000 & July & $\mathrm{D}$ & LIT (4) & $\rightarrow$ DAYLIT & \\
\hline DPB & 2000 & April & D & PEL (4) & $\rightarrow$ DPBPEL & \\
\hline DPB & 2000 & April & $\mathrm{D}$ & $\mathrm{LIT}+(4)$ & $\rightarrow \quad$ DPBLIT + & DPB \\
\hline DPB & 2000 & April & $\mathrm{D}$ & LIT- (4) & $\rightarrow$ DPBLIT- & \\
\hline
\end{tabular}

The online version of the original article can be found at http:// dx.doi.org/10.1007/s00442-004-1711-3

J. Vandekerkhove $(\bowtie) \cdot S$. Declerck $\cdot$ L. Brendonck

L. De Meester

Laboratory of Aquatic Ecology,

Katholieke Universiteit Leuven,

Ch. de Bériotstraat 32,

3000 Leuven, Belgium

E-mail: Jochen.Vandekerkhove@uv.es

Tel: + 32-16-324574

Fax: + 32-16-324575
E. Jeppesen

Department of Freshwater Ecology,

The National Environmental Research Institute,

Vejlsøvej 25, P.O. Box 314, 8600 Silkeborg, Denmark

E. Jeppesen

Department of Plant Ecology, University of Aarhus, Nordlandsvej 68, 8240 Risskov, Denmark

J. M. Conde-Porcuna

Instituto del Auga, Universidad de Granada,

Ramón y Cajal 4 (edif. Fray Luis de Granada),

18071 Granada, Spain 